\title{
http://dx.doi.org/10.35381/racji.v5i1.605
}

\section{Análisis de la adopción frente a la excepcionalidad de la edad entre adoptante y adoptado}

\section{Analysis of adoption versus exceptional age between adopter and adopted}

\author{
Priscila Brigitte Sánchez-Gómez \\ priscila.sanchez@psg.ucacue.edu.ec \\ Universidad Católica de Cuenca, Cuenca \\ Ecuador \\ https://orcid.org/0000-0001-7901-5210 \\ Cecilia Ivonne Narváez-Zurita \\ inarvaez@ucacue.edu.ec \\ Universidad Católica de Cuenca, Cuenca \\ Ecuador \\ https://orcid.org/0000-0002-7437-9880 \\ Cornelio Agustín Borja-Pozo \\ cborjap@ucacue.edu.ec \\ Universidad Católica de Cuenca, Cuenca \\ Ecuador \\ https://orcid.org/0000-0002-6361-2720 \\ Juan Carlos Erazo-Álvarez \\ jcerazo@ucacue.edu.ec \\ Universidad Católica de Cuenca, Cuenca \\ Ecuador \\ https://orcid.org/0000-0001-6480-2270
}

Recibido: 13 de noviembre de 2019

Aprobado: 16 de diciembre de 2019

\section{RESUMEN}

El análisis de la adopción como un proceso garante del Derecho Constitucional del niño, niña y adolescente de pertenecer a una familia y asegurar el cumplimiento de sus derechos establecidos en la Constitución ecuatoriana; sin embargo, la no existencia de la excepcionalidad legal del límite de edad dentro del proceso de adopción cuando existe vínculo afectivo transgrede el derecho constitucional a tener una familia. Atendiendo estas consideraciones, el objetivo del presente artículo 
consiste en analizar la adopción en Ecuador frente a la excepcionalidad de la edad entre los actores como garantía a tener una familia cuando existe vínculo afectivo. Para ello, se empleó una metodología jurídica sustentada en el enfoque cualitativo, y en los métodos histórico lógico, inductivo-deductivo, analítico - sintético. Como principal aporte de la investigación se infiere en la necesidad de reformar el artículo 159 numeral 5 del Código de la Niñez y Adolescencia (CNA).

Descriptores: Adopción; Familia; Vínculo afectivo; Diferencia de edad; Derecho Constitucional.

\section{ABSTRACT}

The analysis of adoption as a guarantor process of the Constitutional Right of the child and adolescent to belong to a family and ensure the fulfillment of their rights established in the Ecuadorian Constitution; However, the non-existence of the legal exceptionality of the age limit within the adoption process when there is an emotional bond violates the constitutional right to have a family. Considering these considerations, the objective of this article is to analyze the adoption in Ecuador against the exceptional age among the actors as a guarantee to have a family when there is an emotional bond. For this, a legal methodology based on the qualitative approach was used, as well as the historical, inductive-deductive, analytical-synthetic historical methods. The main contribution of the research is the need to reform article 159, numeral 5 of the Code of Children and Adolescents (CNA).

Descriptors: Adoption; family; Emotional bond; Age difference; Constitutional right.

\section{INTRODUCCIÓN}

El Derecho Constitucional es garante del establecimiento de los mecanismos de control para el mantenimiento del Estado de Derecho, con la aplicación de las garantías constitucionales en la protección de los derechos fundamentales de los ciudadanos, frente a vulneraciones que afecten a sus derechos y transgreden lo dispuesto en la Constitución. De acuerdo con Raffino (2019), el Derecho Constitucional es "una rama del Derecho Público dedicada al estudio de los principios fundamentales, que determinan la existencia y funcionamiento de un Estado nacional, normalmente establecido en el contenido de una Constitución Nacional o Carta Magna"(párr.1). 
De igual manera, el artículo 11 de la Constitución de la República del Ecuador en su numeral 4 expone: "Ninguna norma jurídica podrá restringir el contenido de los derechos ni de las garantías constitucionales" (p.12). Dentro de este marco, es importante la argumentación de las normas jurídicas, en particular aquellas relacionadas a los derechos fundamentales, lo que permite que más ciudadanos puedan acceder a los beneficios que la constitución otorga para el ejercicio de sus derechos.

A partir de este principio de aplicación de los derechos, se requiere un análisis de la adopción frente a la excepcionalidad de la edad entre adoptante y adoptado, en relación a precautelar los derechos fundamentales, siendo uno de ellos, el principio del interés superior del niño (PIN), de tal forma que se logren garantizar el derecho a tener una familia permanente y definitiva. Es importante reconocer a la adopción como un proceso garante del derecho constitucional del niño, niña, adolescente o joven de pertenecer a una familia no biológica de conformidad con la ley, donde el adoptante y adoptado tienen los mismas derechos y deberes que impone la patria potestad a los progenitores (Código de la Niñez y Adolescencia, 2003).

En este sentido, el problema central que se analiza en este artículo hace referencia al derecho a tener una familia a través de la adopción y la flexibilización de la edad entre adoptante y adoptado en el proceso de adopción, con el objeto de que al calificar la idoneidad de los solicitantes de adopción realizado por las Unidades Técnicas de Adopciones del Ministerio de Inclusión Económica y Social, se cumpla con los parámetros establecidos en el artículo 159 del Código de la Niñez y Adolescencia, al mismo tiempo, identificando excepcionalidades para la adopción con respeto al límite de la diferencia de edad: adopción del hijo del cónyuge, adopción de parientes donde claramente se garantiza a través de esas excepcionalidades el derecho a tener una familia sin tomar en cuenta la limitación de la diferencia de la edad entre adoptante y adoptado.

A este respecto, la restricción para los solicitantes de acceder al proceso de adopción por no cumplir con los requisitos de la diferencia de edad entre adoptante y adoptado cuando existe vínculo afectivo al no estar estos casos concretos 
claramente expresado dentro de las excepciones establecidas en el artículo 159 numeral 5, en concordancia con el artículo 157 del Código de la Niñez y Adolescencia, vulnera el derecho constitucional a tener una familia a través de la adopción (Constitución de la República del Ecuador, 2008).

En consecuencia, para que el Estado pueda brindar las condiciones y acciones necesarias que garantice la tutela efectiva para la restitución del derecho del niño, niña, adolescente o joven a pertenecer a una familia, este artículo centra su objetivo en analizar la adopción en el Ecuador frente a la excepcionalidad de la edad entre adoptante y adoptado como garantía a tener una familia cuando existe vínculo afectivo, considerando que este derecho y principio constitucional no puede ser vulnerado, más aún si tienen atención preferente por parte del Estado al pertenecer los niños, niñas, adolescentes y jóvenes a los grupo de atención prioritaria establecido en la Constitución de la República del Ecuador, quienes en algunos casos presentan doble vulnerabilidad, situación que se reduce a la siguiente pregunta de investigación: ¿Cuáles son los derechos que se presumen vulnerados en la adopción de niños, niñas, adolescentes y jóvenes con vínculo afectivo frente a la falta de excepcionalidad de la limitación de la diferencia de edad entre adoptante y adoptado contemplado en el Artículo 159 numeral 5 del Código de la Niñez y Adolescencia?

\section{DESARROLLO}

\section{Principios jurídicos generales sobre la adopción}

La adopción, es un concepto dinámico, cuyo desarrollo evolutivo a nivel mundial ha respondido a las necesidades de cada lugar y realidad social de la época. Cabe considerar los instrumentos internacionales sobre la adopción que desde la perspectiva general se encuentran consagrados en la Declaración de Ginebra que fue creada en 1949, para reconocer los derechos específicos para los niños y niñas, a pesar de ser el primer texto histórico sobre derechos sociales de la niñez y protección de la infancia, más no de derechos civiles haciendo que pierda fuerza vinculante para los Estados, más tarde llegaría la Convención sobre los Derechos 
del Niño (CIDN), que data del año 1989, a través de la cual se enfatiza la igualdad de derechos en los niños y los adultos, permitiendo a los Estados que se adhieren a la convención y se comprometen a cumplirla para lograr que cada niño goce plenamente sus derechos estipulados en articulado 3 donde menciona el principio de interés superior del niño (ISN), frente a las diversas situaciones que puedan vulnerar sus derechos fundamentales (Convención sobre los Derechos del Niño, 1989).

Ecuador en el año 1990 aprobó la Convención Internacional de los Derechos del Niño y, con ello la necesidad de reformar la legislación en el ámbito de menores y de familia; el Código de Menores fue un instrumento novedoso por el nuevo sentido que se le dio a temas como: necesidad de considerar el ISN en la toma de cualquier medida administrativa, legislativa o judicial; observar su origen étnico o cultural; obligación de tomar en cuenta su opinión; reconocer a la familia, la comunidad y sociedad conjuntamente con el Estado como garante de la aplicación de los derechos de los menores (Código de Menores, 1992).

La adopción como una garantía de protección en Ecuador fue aprobada en el Registro Oficial número 86 del 15 de diciembre de 1948, aquí se decretan 19 artículos que hablan sobre la institución de la adopción de menores, quienes pasan a formar parte de una familia que no lo es, como suya. La primera institución que se encargó de los procesos de adopción en Ecuador fue el Instituto Nacional del Niño y la Familia (INNFA), institución que tenía como objetivo la atención a la niñez y a la familia mediante la asistencia social y médica con el fin de lograr el bienestar de los mismos; esta institución tiene su origen por decreto ejecutivo número 175 del año 1960, decreto que da inicio al Patronato Nacional del Niño del Ecuador (Ferrín \& San Andres, 2013).

En 1993 se instaura, la Convención de la Haya para la protección de los Niños y Cooperación en materia de adopciones entre países, con el fin de construir una adopción plena y asegurar la protección de los intereses de los niños, padres biológicos y padres adoptivos involucrados en el proceso de adopción entre países, este proceso aplica cuando los candidatos a adoptantes tienen su domicilio en otro Estado que haya suscrito al mismo convenio en materia de adopción internacional 
vigente con Ecuador. Este sistema de cooperación entre países de origen y recepción, asegura la adopción internacional y de esta manera logra ser una ventaja de otorgar una familia permanente a niños, niñas y adolescentes (NNA) que no tuvieron las posibilidades de colocación en su Estado de origen. La adopción internacional es una alternativa más que crea las condiciones que garantizan los procesos de adopción (Convenio de la Haya sobre Adopción, 1993).

En 1998 la Asamblea Nacional incorporo una serie de reformas en pro de los NNA en la Constitución de la República del Ecuador (CRE), siendo esta Constitución la décima novena que abrazo el país al proveerle de normas propias y jurisdicción especial para los NNA, diferenciando a los menores de edad de los adultos (Erazo, 2014). Actualmente la CRE del año 2008 cuenta con un capítulo de derechos que benefician a los NNA.

En Ecuador el Código Civil vigente en su artículo 314 define a la adopción como una institución en la que el adoptante adquiere derechos y obligaciones, conforme lo establece el artículo 45 de la CRE, en donde el Estado reconoce y garantiza a los NNA, el efectivo goce de los derechos comunes del ser humano como salud integral, educación y seguridad social, a tener una familia y disfrutar de la convivencia familiar (Código Civil Ecuatoriano, 2005). El CNA expedido en el año 2003, en su artículo 1 expresa la finalidad de garantizar a todos los NNA ecuatorianos sus derechos fundamentales conforme al principio del interés superior de la niñez y adolescencia, en su artículo 153 establece aspectos formales para iniciar un proceso de adopción, la priorización de la adopción nacional sobre la internacional, el perfil de parejas legalmente constituidas, grado consanguinidad y la idoneidad de los candidatos a adoptantes, al mismo tiempo en su artículo 158 manifiesta la aptitud legal del NNA para ser adoptado.

Estas situaciones son la orfandad respecto de ambos progenitores o si se desconoce quiénes son, si existe o no privación de la patria potestad y según el caso el consentimiento de uno o ambos progenitores, luego de la declaración judicial de adoptabilidad del niño, niña, adolescente o joven se debe seguir el debido proceso y 
por consiguiente llevar a cabo el respectivo procedimiento administrativo y judicial de adopción (Código de la Niñez y Adolescencia, 2003).

Dentro de los procedimientos administrativos y judiciales de la adopción los requisitos establecidos en el artículo 159 del CNA se identifican elementos tales como el domicilio, la legalidad de los adoptantes, edad referencial de 25 años, las limitaciones de la edad, buen estado de salud física y mental, disposición de recursos económicos y la no tenencia de antecedentes penales (Código de la Niñez y Adolescencia, 2003). Al respecto, es necesario considerar el límite de edad entre adoptado y adoptante con el objeto de evidenciar los factores legales que dificultan el proceso de adopción de los NNA y jóvenes a ser adoptados, ya que pone en riesgo la garantía de los derechos reconocidos en la CRE y atenta contra la consecución de la adopción, lo que genera una problemática con incidencia social y jurídica, causando prejuicios en ambos actores, vulnerando la posibilidad de conformar una nueva familia e instituyendo así el proceso de adopción, como un peligro dentro del sistema de protección ecuatoriano (Tamayo Vásquez \& Pino Loza, 2019).

Actualmente, en el Ecuador la adopción es un acto jurídico, es una institución que establece vínculos de parentesco civil propios de la relación parento filial con condiciones que generan un auténtico bienestar del menor y del respeto de sus derechos (Ibañez, 2006). En este sentido, la adopción es una institución que dentro de cada ordenamiento jurídico al cual se circunscriba el Estado, crea para el adoptado y adoptantes lazos filiales voluntarios, obligaciones y derechos para ambas partes (Sentencia C-814/01, 2001). Como complemento de lo anteriormente señalado se indica así mismo a la adopción como instrumento viable para la inserción inmediata del NNA a una familia, donde prima no solamente derechos y deberes de los adoptantes y adoptados sino también el vínculo afectivo (Ortega, 2015).Como resultado de estas definiciones se puede colegir al proceso de adopción como un acto jurídico que permite a un NNA o joven a vivir en familia garantizándole de esta manera el efectivo ejercicio de su derecho constitucional. 
Una vez definido el concepto de adopción, resulta interesante destacar las fases de la adopción dentro del sistema ecuatoriano; como son: la fase administrativa y la fase judicial, establecidos en el CNA, las mismas que se encargan del bienestar del menor desde el inicio del proceso. La fase administrativa, está a cargo Ministerio de Inclusión Económico y Social (MIES), a través de las Unidades Técnicas de Adopciones (UTA) y los Comités de asignación familiar quienes ejecutan el efectivo ejercicio de pertenecer a una familia, cuya finalidad es determinar una familia idónea y definitiva al niño, niña y adolescente en condición legal de ser adoptado, apoyado en el artículo 165 del CNA, que menciona el objeto de la fase administrativa en lo referente a la situación óptima para ser sujeto de adopción y de idoneidad de él o los adoptantes, además emitir la resolución administrativa de la asignación de una familia a un NNA o joven (Ministerio de Inclusión Económica y Social, 2019).

Una vez superada la fase administrativa, la misma termina con el informe positivo de emparentamiento establecida en el CNA en concordancia con el Protocolo para el Proceso de Emparentamiento (Dirección Nacional de Adopciones y Unidades Técnica de Adopciones Regionales, 2012). En la actualidad el proceso de emparentamiento se lo conoce como proceso de apego y vinculación afectiva del NNA o joven. De ser positivo este proceso se da inicio a la fase judicial de la adopción que consiste en la concurrencia de las partes ante el Juez de la Unidad de Familia Niñez y Adolescencia del Cantón del domicilio donde resida el NNA o joven para que, por medio de la sentencia el Juez conceda la adopción nacional o internacional plena al NNA o joven adoptado, según corresponda (Ministerio de Inclusión Económica y Social, 2019).

\section{Análisis de la excepcionalidad de la edad en el caso de vínculo afectivo}

Es importante mencionar que, en el articulo157 del CNA, se hace referencia sobre la edad del adoptado; es decir, la adopción en Ecuador es posible para personas que no han cumplido la mayoría de edad y, en ciertos casos concretos se permite la adopción hasta los veintiún años. En el artículo 159 numeral 5 ibídem se encuentra una regla relativa a la edad que deben cumplir los solicitantes de adopción, 
incluyendo dos excepcionalidades; sin embargo, hay interesados en iniciar un proceso de adopción por la existencia de apego y vínculo afectivo que no se enmarcan dentro de las excepcionalidades (Véliz Valencia, 2019).

Muy poco se ha estudiado sobre la adopción excepcional por vínculo afectivo, por lo que no existe un verdadero criterio doctrinario que permita el reconocimiento de lazos familiares preexistentes sin ser pariente o hijo de cónyuge. A este respecto, Mcwhorter (2004) refire que existen casos de NNA y jóvenes que han generado vinculo parental con personas sin línea de consanguinidad, sugiriendo para este caso la adopción de adultos sin tomar en cuenta el límite de diferencia de edad entre adoptante y adoptado como un mecanismo perfecto para legalizar los vínculos existentes. En consecuencia, la adopción es el fin de una relación familiar de apego y vínculo afectivo de hecho donde se ha incorporado a un NNA y joven a una familia, dándole por años el tratamiento de hijo.

La diferencia de edad que debe existir entre adoptante y adoptado fue previamente analizado por el legislador en el Ecuador, quién consideró la necesidad de generar excepcionalidades frente a la adopción con el objeto de garantizar el derecho constitucional a tener una familia, otorgando la facultad para calificar y declarar la idoneidad de una familia solicitante de adopción a la UTA, sin embargo, el legislador en el Ecuador no consideró dentro de las excepcionalidades para la adopción cuando se trata de calificar a solicitantes que tienen apego y vínculo afectivo con NNA y jóvenes que sin ser parientes, ni hijo del cónyuge desean realizar el proceso de adopción. Zannoni (1978) afirma que la diferencia de edad entre adoptante y adoptado tiene como fin que la adopción pueda ser ejercida por personas que tengan madurez afectiva y humana, para tal efecto, de suscitarse casos concretos y excepcionales como el que se analiza en este artículo, estaría más que demostrado la madurez, responsabilidad y amor que fue otorgado a NNA y jóvenes por lo que no cabría la limitación de la diferencia de edad y en todo caso esas particularidades deberían ser resueltas por los jueces.

La legalización de una adopción por apego y vínculo afectivo se puede efectivizar, cuando exista pleno conocimiento y valoración del legislador para reconocer el 
afecto generado entre adoptante y adoptado, debiendo entonces claramente dejar sentado como excepcionalidad los casos de adopción donde existan relaciones que hayan compartido durante años. En esta misma línea de pensamiento, Méndez ( 2001) se refiere a la adopción como aquella que reconoce y protege jurídicamente las relaciones afectivas que existe entre los actores del proceso.

Señalar que en todos los casos de adopción se debe dar un tratamiento excepcional es falso, las excepcionalidades surgen cuando del antecedente de historia de vida de los NNA y jóvenes se encuentran casos de: acogimiento institucional, acogimiento familiar (AF), abandono con padrinos o con personas que no son parientes por prolongados períodos de tiempo, desencadenando esta situación en generación de apego y vínculos afectivos seguros que no fueron previstos por las partes involucradas. Según Bowlby (1979) considera que toda persona nace y muere con una tendencia a establecer vínculos afectivos con otras personas con el fin de sentirse protegido, apoyado y confortado; en consecuencia, los programas de AF apuntan a cumplir con el derecho del NNA a vivir en familia, cubriendo sus necesidades no solo de sustento y de cuidado, sino también sus necesidades emocionales que le genera apego y vínculo con la o las personas que le guían en su crecimiento hasta desear ser adoptado o adoptada por las personas que estuvieron a cargo de su cuidado.

En este contexto, la Corte Constitucional Colombiana ha señalado que la institución familia va más allá del reconocimiento por lazos consanguíneos, la familia está en la vinculación afectiva, sin importar las personas que la integran, el concepto de familia no es cerrado, se instaura a base de amor, respeto y solidaridad (Corte Constitucional de Colombia, 2011). Para tal efecto, no se podría negar a un NNA o joven y a su familia acogiente a realizar el proceso de adopción por no cumplir el parámetro legal de la edad. Es importante reconocer que la adopción genera vinculo similar al que se deriva de la filiación (Belluscio, 2011).

A este respecto es necesario señalar que en la oficina de la UTA del MIES Zona 6 acude un alto índice de solicitantes de adopción que desean adoptar a un niño, niña, adolescente o joven menor de 21 años con el que mantienen vínculo afectivo por 
largos periodo de tiempo, con quienes comparten el hogar, los solicitantes y quienes han asumido los deberes y obligaciones que conlleva la patria potestad como si se tratara de su hijo o hija, diferenciando el un caso con el otro el origen que generó ese vínculo afectivo.

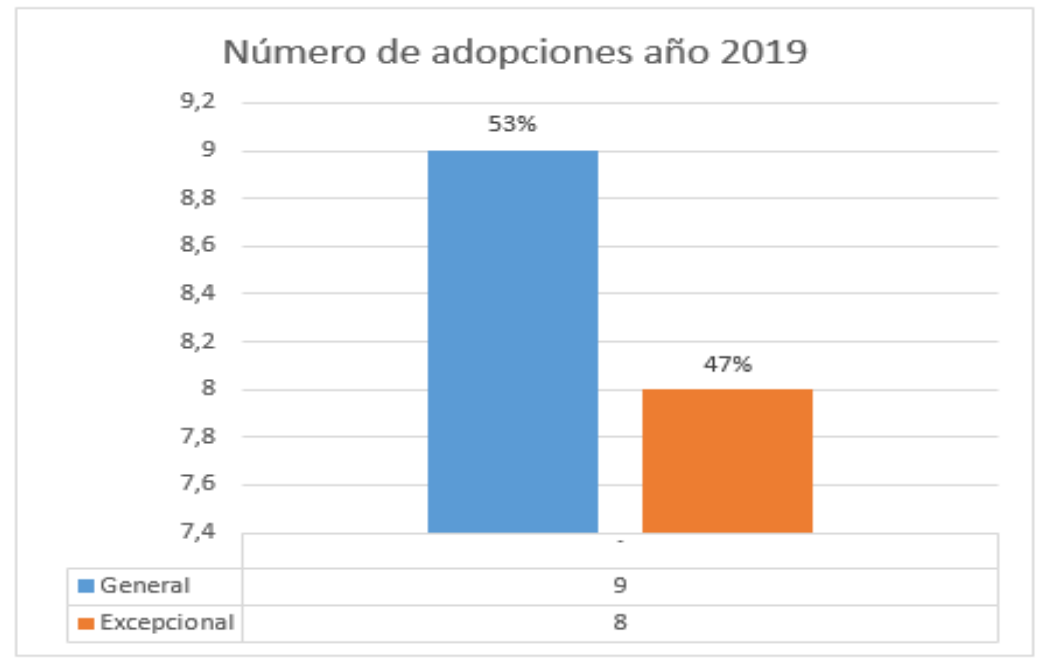

Figura 1. Adopciones durante el año 2019

De estas evidencias, se determina que, de los 17 procesos administrativos de adopción que se llevó a cabo en la zona 6 en el año 2019; 9 adopciones fueron procesos generales cumpliendo los requisitos establecidos en el articuloArt. 159 del CNA, en concordancia con el Manual de Proceso Gestión de Adopciones Nacionales y, 8 adopciones fueron excepcionales.

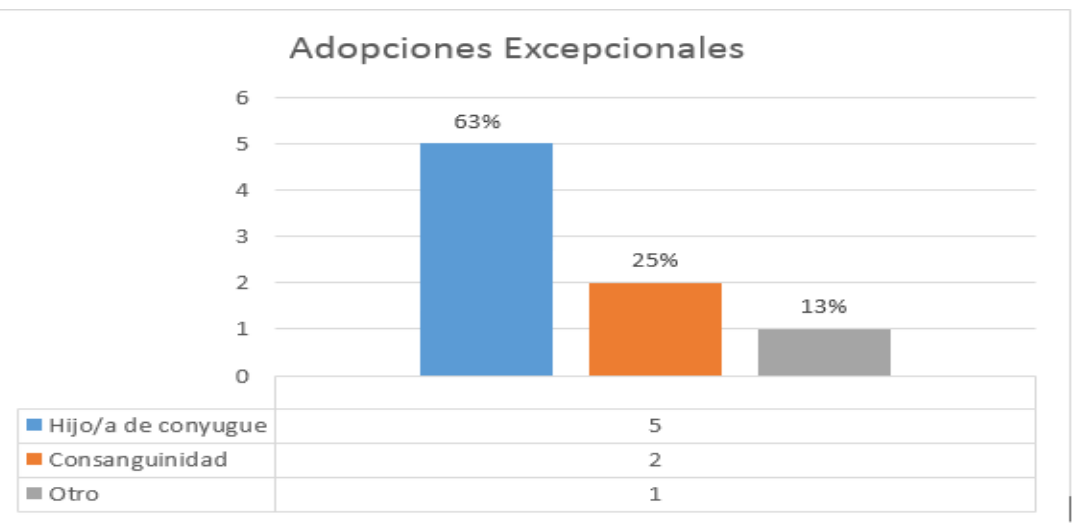

Figura 2. Adopciones excepcionales 
De los datos de la figura 2, se puede observar que de las 8 adopciones excepcionales que se llevo a cabo en el año 2019, 5 procesos de adopción fueron adopción del hijo del conyuge, cumpliendo para ello los 9 requisitos establecidos en el articulo 159 del Código de la Niñez y Adolescencia, incluyendo la excepcionalidad prevista en el numeral 5; 2 procesos de adopción se dieron por línea de parentezco ( hermana paterna y abuela materna) en 1 de los 2 casos presentados se empleó la excepcioanlidad establecida en el Art. 159 numeral 5 del Código de la Niñez y Adolescencia; es decir, no se aplicó las limitaciones de la edad; y finalmente se conto con 1 proceso de AF sin vinculo consanguineo, donde el niño ha compartido el hogar de la solicitante por mas de 10 años y la interesada no cumple con el requisito de la diferencia de edad entre adoptante y adoptado (tener una diferencia de edad no mayor de 45 años con el adoptado) establecido en el numeral 5.

\section{Derecho constitucional a tener una familia: referencia a la adopción}

Para Angulo (2015) el núcleo que permite la conformación de una verdadera sociedad es la familia y todos deben formar parte de ella por derecho desde la infancia, sobre todo con la prioridad del ISN. Así mismo, el Estado debe brindar protección a la familia en la prevención de afectaciones contra los diversos tipos de familia que no cumplen el esquema conservador de estereotipos instaurados en la sociedad. La institución de la adopción es una garantía de derechos para NNA en la orfandad y con el objetivo de ofrecer el cumplimiento del pleno goce de los derechos reconocidos en la CRE de convivir con una familia, Gómez (2015), expresa que la declaración de adoptabilidad debe obedecer siempre al ISN garantizando su integración familiar, entorno social favorable y excelentes condiciones de educación, entro otros aspectos religiosos y culturales.

Existen nuevas formas de organización familiar, Navarro (2013), presenta su noción de los tipos de familia en relación familiar biológica o adoptiva con vínculos jurídicos plenamente establecidos y a su vez describe un elemento subjetivo expresado en la protección frente a valores individuales del niño, niña y adolescente, la misma que define vínculos morales para una convivencia social. En la CRE, en el artículo 67 se 
reconoce a la familia en sus diversos tipos familia según lo planteado en la investigación de Martínez (2019) pudo determinar familias de padres separados, monoparentales, reconstruidas, homoparentales y nuclear, no siendo el vínculo matrimonial la única fuente de familia como parejas del mismo sexo, sin embargo, en su artículo 68 la adopción corresponderá solo a parejas de distinto sexo, lo cual origina una desigualdad jurídica para la adopción NNA, discriminando a la persona con orientación sexual diferente.

En Ecuador existe un alto nivel de menores en estado de abandono y demás situaciones en donde puede existir la vulneración de los derechos fundamentales a tener una familia, según lo detalla Padilla, (2011), el principio del ISN hace referencia a que los NNA se les garantice las condiciones necesarias para el pleno goce de sus derechos, no obstante, cuando se presentan conflictos jurídicos al momento de aplicar el principio del ISN a pertenecer a una familia, siempre debe primar el derecho del menor, para ello se analizarán todos los casos donde se evidencien vulneración de derechos, de ahí que la posición de la Corte Constitucional luego del estudio de casos concretos efectuará la aplicación de este principio, preservando el equilibrio entre los derechos del niño y el de los padres al mismo tiempo que garantiza el interés jurídico supremo al desarrollo integral del NNA. Por consiguiente, determina una línea jurisprudencial para identificar los precedentes establecidos en la inmediata aplicación del principio del ISN y así proteger su derecho a tener una familia y a permanecer con ella, tal como lo plantea la CDN, en donde los Estados que la integran se comprometen a velarán por el ISN, y la familia brindar las condiciones óptimas para que crezcan en un ambiente sano.

A partir de esta afirmación, se expone que no se puede determinar una norma exacta sobre la armonización de los derechos contrapuestos de los menores y padres cuando no se garantiza el derecho a tener una familia y no ser desvinculado de ella, ante ello y según el caso concreto se ha de tener como primacía que el ejercicio de los derechos de los padres no pueden poner en riesgo los intereses prevalecientes del menor. 
El artículo 3 de la Convención sobre los Derechos del Niño del año 1989, insta a que todas las instituciones públicas, privadas, autoridades administrativas y órganos legislativos que actúen frente a situaciones en las que estén en juego derechos de los NNA, aplicando el ISN. Otro elemento, que respalda la situación descrita es la CRE en su artículo 44, en donde el Estado, sociedad y la familia atenderán prioritariamente el ISN sobre las demás personas. Teniendo en cuenta lo expuesto, se establece la prevalencia de los derechos de la infancia sobre los derechos de los demás y que el Estado es el garante para que se cumplan las disposiciones legales o administrativas para la aplicación de la norma que favorezca el ISN.

La actual CRE, en su artículo 169 señala siete numerales que reconocen a la familia como el pilar fundamental de toda la sociedad, donde los derechos de los NNA, deben ser respetados e invocados cada vez que exista vulneración a sus derechos, por estar declarados y precautelados en la Constitución. La CRE menciona que serán parte de los demás miembros de la familia, los niños biológicos como los adoptados. Bajo esta consideración, se trata de lograr una familia idónea para un NNA que legalmente ha conseguido ser declarado apto para adopción.

\section{METODOLOGÍA}

En el presente artículo se aplicó la investigación histórico-lógico, a través del cual, se realizó un estudio en base a la evolución de la adopción desde sus inicios hasta la actualidad, de igual manera, se empleó la investigación bibliográfica que proporcionó el conocimiento de las investigaciones existentes sobre la adopción y la excepcionalidad del límite de edad. El método inductivo - deductivo facilitó analizar el problema de forma particular y luego generalizarlo a partir de la revisión de los expedientes de solicitantes de adopción, del mismo modo, se recurrió al método analítico-sintético al descomponer los requisitos que cumplen los solicitantes de adopción relacionado a la edad de los NNA adoptados, observar las causas, la naturaleza y los efectos que ocasionaría sino se efectivizara la adopción. El Universo de estudio estuvo conformado por 17 expedientes presentados en la Unidad Técnica 
de Adopciones del MIES Zona 6, con sede en la Ciudad de Cuenca durante el año 2019 (Rojas, Erazo, Pozo y Narváez, 2020).

\section{APORTES GENERADOS}

La institución de la adopción ha ido evolucionando a través del tiempo, pasando de la forma de proveer una descendencia a una familia, hasta llegar a la actualidad como una forma de proveer una familia idónea al niño, niña, adolescente o joven menor de 21 años que carece de ella por diferentes motivos.

El límite de la diferencia de edad entre los que desean adoptar y ser adoptados marcados en la ley no debería ser un obstáculo para garantizar el Derecho Constitucional a tener una familia. La hipótesis del apego citada por Bowlby (1979) ayuda a entender que un vínculo seguro con un cuidador donde la relación sea estable y permanente, permite un adecuado desarrollo cognitivo y mental del niño que llegará a ser adulto.

Actualmente existen varios estudios sobre el vínculo seguro y la capacidad de fortaleza frente a eventos estresantes o de pérdida. Estamos presente en un mundo cambiante en que las pérdidas ocurren; las familias se divorcian, y alguno de los progenitores fallecen o se alejan. De igual manera sucede con los casos de NNA que han sido abandonados y que han sido entregados en acogimiento familiar con orden judicial, sin que la autoridad judicial haya prevenido que la familia acogiente cumpla con una edad que a futuro no interfiera como obstáculo para lograr la adopción del NNA que tiene a su cuidado y protección de no ser posible la reinserción familiar.

El artículo 169 de la CRE reconoce a la familia como la piedra angular de toda la sociedad, donde los derechos de los NNA, deben ser respetados e invocados cada vez que exista vulneración a sus derechos, los mismos que se encuentran reconocidos y garantizados. Además, reconoce que serán parte de los demás miembros de la familia, los niños biológicos como los adoptados. En tal virtud, se trata de lograr una familia idónea para un NNA que legalmente se encuentre en condiciones de adoptar. 
De la investigación realizada en la UTA Zonal 6 se obtiene información respecto a la existencia de casos de niños, niñas y adolescentes que han acudido a esta dependencia administrativa sin dar sus datos de identificación o contacto y exponen que desean ser adoptados por la familia acogiente o por la o las personas que les cuidaron $y$, que revisado los requisitos legales para adoptar los solicitantes no cumplen con la formalidad del límite de edad que debe existir entre los actores, de acuerdo al artículo 159 numeral 5 del CNA, procediendo al saber que no hay una excepcionalidad para estos casos de vínculo afectivo a retirarse sin presentar formalmente su petición, para que la UTA pueda elevar a consulta al Organismo competente o dar la respuesta por escrito y de manera motivada, para que el mismo sirva de antecedente para los casos venideros.

De acuerdo al artículo 159 numeral 5 del CNA, los solicitantes deben cumplir requisitos de forma: la diferencia de edad entre ellos, la misma que tiene límites para que proceda, y al no cumplir con estos parámetros legales no podría darse la adopción, de allí la necesidad de proponer una reforma al CNA en el artículo 159 numeral 5 que incorpore otra excepcionalidad a más de las dos existentes en los siguientes casos: cuando el niño, niña, adolescente y joven ha estado integrado en el hogar de los solicitantes en calidad de AF o cuando los niños, niñas y adolescentes se encuentran compartiendo el hogar de los candidatos a adoptantes desde su niñez por un periodo no inferior a cuatro año, basados en las excepcionalidades para los casos de adopción de mayores de 18 años de edad por el vínculo que generaron este tipo de relaciones (Ver figura 3). 


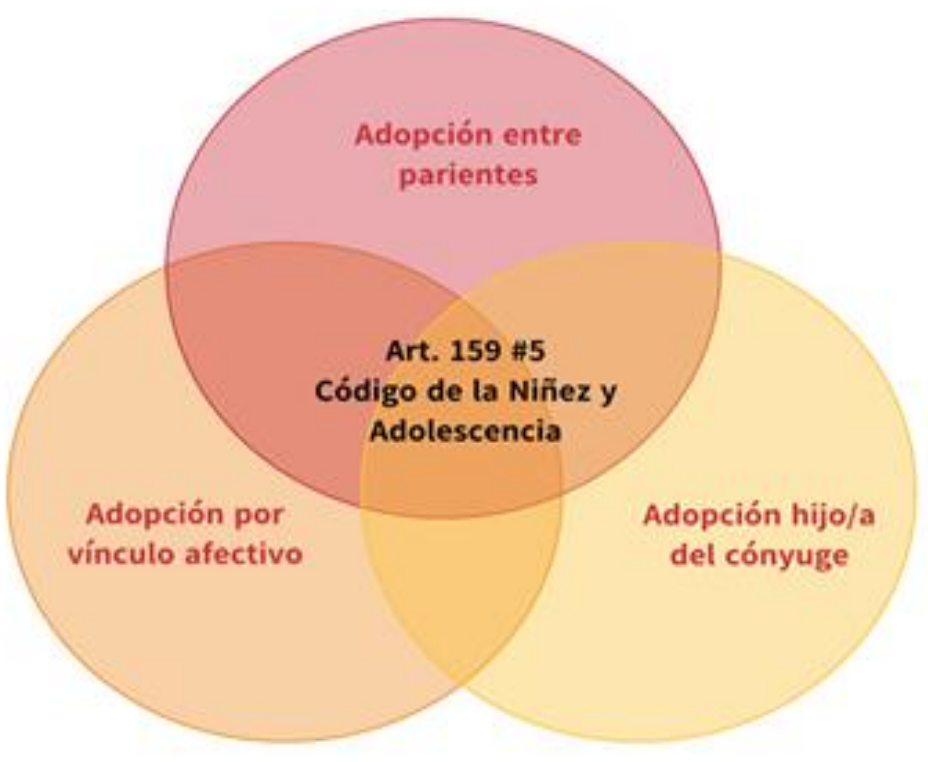

Figura 3. Excepcionalidades en el artículo 159 numeral 5 en los que no se aplicará la diferencia de edad entre adoptante y adoptado

\section{REFERENCIAS CONSULTADAS}

1. Angulo, T. (2015). Derecho a la protección de la familia. Perfiles de las Ciencias Sociales, 3(5).

2. Belluscio, A. (2011). Manual de Derecho de Familia. Buenos Aires, Argentina: AbeledoPerrtp S.A.

3. Bowlby, J. (1979). The Making and Breaking of Affectional Bonds. London, Inglaterra.

4. Código Civil Ecuatoriano (2005). Ecuador. Recuperado de https://www.quito.gob.ec/lotaip2013/a/CodigoCivil2005.pdf

5. Código de Menores (1992). Registro Oficial número 995. Recuperado de https://www.etapa.net.ec/Portals/0/TRANSPARENCIA/Literal-a2/CODIGO-DELA-NINEZ-Y-ADOLESCENCIA.pdf

6. Código de la Niñez y Adolescencia (2003). VII De la Adopción. Publicado en el Registro Oficial. Quito, Ecuador. Recuperado de 
https://www.etapa.net.ec/Portals/0/TRANSPARENCIA/Literal-a2/CODIGO-DELA-NINEZ-Y-ADOLESCENCIA.pdf

7. Constitución de la República del Ecuador (2008). Quito, Ecuador: Registro Oficial.

https://www.oas.org/juridico/pdfs/mesicic4_ecu_const.pdf

8. Convención sobre los Derechos del Niño (1989).Recuperado de http://plataformadeinfancia.org/derechos-de-infancia/la-convencion-de-losderechos-de-lainfancia/?gclid=Cj0KCQiApt_xBRDxARIsAAMUMu8nBr9kKIkHzf5j2jtUKfLPLnKIFRwR36L_G1rSXB3nAI0nYfNM3EaAtjgEALw_wcB

9. Convenio de la Haya sobre Adopción (1993). Convenio relativo a la protección del niño y a la cooperación en materia de adopción internacional Recuperado de https://www.oas.org/dil/esp/Convenio_Haya_Proteccion_del_Nino_Cooperaci on_en_Materia_Adopcion_Internacional_Espana.pdf

10. Corte Constitucional de Colombia (2011). Sentencia C-577-11. Recuperado de https://www.corteconstitucional.gov.co/relatoria/2011/C-577-11.htm

11. Corte Constitucional de Colombia (2001). Sentencia C-814/01. Recuperado de https://www.corteconstitucional.gov.co/relatoria/2001/c-814-01.htm

12. Dirección Nacional de Adopciones y Unidades Técnica de Adopciones Regionales. (2012). Recuperado de https://www.inclusion.gob.ec/la-adopciones-vivir-la-experiencia-de-disfrutar-el-tener-un-hijao/

13. Erazo, G. (2014). Eficacia en el proceso de adopción del menor de edad en el marco legal ecuatoriano. Quito, Ecuador.

14. Gómez, B. (2012). Adoptabilidad: el derecho del niño/a a vivir en familia. Scripta Nova. Revista Electrónica de Geografía y Ciencias Sociales, 16(395).

15. Ibañez, A. (2006). La adopción: el interes superior del menor frente a los derechos subjetivos públicos de terceros. Popocatépetl, Mexico.

16. Martínez, R. (2014). La adopción de personas mayores de edad en sede notarial Universidad Regional Autónoma de los Andes. Facultad de Jurisprudencia. Recuperado del 23 de enero de 2020 de: http://dspace.uniandes.edu.ec/bitstream/123456789/3189/1/TUAMDN0032014.pdf 
17. Mcwhorter, S. (2004). The complete adoption and fertility legal guide. Naperville.

18. Méndez, D. (2001). Derecho de Familia (Vol. III). (Rubinzal, Ed.) Buenos Aires, Argentina.

19. Ministerio de Inclusión Económica y Social. (2019). Manual de procesos de la gestión de adopciones nacionales. Publicado en el Registro Oficial Edición Especial 123. Quito, Ecuador.

20. Navarro, R. (2013). Repositorio de recursos de Aprendizaje. Recuperado de http://brd.unid.edu.mx/la-familia-comoespacio-socializador-de-la-infancia/

21. Ortega, J. (2015). La Celeridad en los trámites admibnistrativos y judicuiales en el proceso de adopción de niños, niñas y adolescentes y los derechos de repación de los solicitantes. Babahoyo, Ecuador.

22. Padilla, S. (2011). Aplicación del principio del interés superior del niño (a) como mecanismo para proteger el derecho de los niños y las niñas a tener una familia y a no ser separados de ella. Universidad Externado de Colombia, Bogotá D.C., Colombia.

23. Rojas Valdivieso, M. C., Erazo Álvarez, J. C., Pozo Cabrera, E. E., \& Narváez Zurita, C. I. (2020). Prueba en Garantías Jurisdiccionales. Falta de regulación y afección al derecho a la defensa y libertad probatoria en el Ecuador. Iustitia Socialis, 44.

24. Ferrín, R., \& San Andrés, M. (2013). El proceso de adopción en el ecuador debe contribuir eficazmente al cumplimiento de los derechos de los niños, niñas y adolescentes previstos en la constitución y propuesta de Reforma Titulo VII Código de niñez y adolescencia para viabilizar el trámite. Recuperado de http://repositorio.uees.edu.ec/handle/123456789/105.

25. Raffino, M. (2019). Derecho Constitucional. Argentina: Concepto. Recuperado de https://concepto.de/derecho-constitucional/

26. Zannoni, E. (1978). Derecho de familia (1 ed. ed.). Buenos Aires, Argentina: Astrea.

(C2020 por los autores. Este artículo es de acceso abierto y distribuido según los términos y condiciones de la licencia Creative Commons Atribución-NoComercial-Compartirlgual 4.0 Internacional (CC BY-NC-SA 4.0) 